\title{
Lifetime maps for orbits around Callisto using a double-averaged model
}

\author{
Josué Cardoso dos Santos ${ }^{1} \cdot$ Jean P.S. Carvalho ${ }^{2} \cdot$ Antônio F.B.A. Prado ${ }^{3} \cdot$ Rodolpho Vilhena de Moraes $^{4}$
}

Received: 8 January 2017 / Accepted: 26 October 2017 / Published online: 20 November 2017

(c) Springer Science+Business Media B.V., part of Springer Nature 2017

\begin{abstract}
The present paper studies the lifetime of orbits around a moon that is in orbit around its mother planet. In the context of the inner restricted three-body problem, the dynamical model considered in the present study uses the double-averaged dynamics of a spacecraft moving around a moon under the gravitational pulling of a disturbing third body in an elliptical orbit. The non-uniform distribution of the mass of the moon is also considered. Applications are performed using numerical experiments for the Callistospacecraft-Jupiter system, and lifetime maps for different values of the eccentricity of the disturbing body (Jupiter) are presented, in order to investigate the role of this parameter in these maps. The idea is to simulate a system with the same physical parameters as the Jupiter-Callisto system, but with larger eccentricities. These maps are also useful for validation and improvements in the results available in the literature, such as to find conditions to extend the available time for a massless orbiting body to be in highly inclined orbits under gravitational disturbances coming from the other bodies of the system.
\end{abstract}

Keywords Lifetime of orbits - Double-average - Third-body perturbation $\cdot$ Orbital perturbations $\cdot$ Moons, perturbation maps

$凶$ J. Cardoso dos Santos

josuesantosunesp@gmail.com

1 São Paulo State University (UNESP), Guaratinguetá 12516410 , Brazil

2 Universidade Federal do Recôncavo da Bahia (UFRB), Feira de Santana 44085132, Brazil

3 National Institute for Space Research (INPE), São José dos Campos 12201970, Brazil

4 Federal University of São Paulo (UNIFESP), São José dos Campos 12231280, Brazil

\section{Introduction}

The present study deals with the problem of using lifetime maps for a qualitative study of a dynamical system that represents third-body effects in the context of the restricted three-body problem with an inner massless body in an eccentric orbit. The study also intends to add the effects of the gravitational field due to a central body with ellipsoidal shape ( $J_{2}$ and $C_{22}$ ), i.e., with a non-uniform gravitational potential, in order to improve the analysis and make this investigation helpful for further analysis such as planning of space missions. For practical purposes, an application of the model which represents the physical forces mentioned previously is considered to study the motion of a spacecraft that is assumed to be an inner body that is orbiting Callisto in low-altitude orbits $(\sim 100 \mathrm{~km})$. Callisto is Jupiter's fourth Galilean moon, and it is used as the central body of our system. The spacecraft orbiting this moon suffers the gravitational pulling of Jupiter, considered as the external (outer) disturbing body of the system. Indeed, since Jupiter is a very massive disturbing body, the Kozai-Lidov effects appear for orbits with high inclinations (Prado 2003), which is a dynamical behavior first discussed in the work of Kozai (1962) and Lidov (1962) in the framework of the quadrupolar secular restricted three-body problem considering a massless particle as the inner body of the system. This particle is assumed to be orbiting a massive central body under the gravitational attraction of a massive external third body.

It is important to mention that the quadrupolar secular restricted three-body problem presents two cases, which are the inner and the outer restricted case. In the inner restricted case, which corresponds to the dynamical architecture of the present study, the conservation of the normal component of the angular momentum enables the inner particle to periodically exchange its eccentricity with inclination, 
which is known as the Kozai-Lidov mechanism. The "inner" restricted three-body problem, as it is sometimes called (Farago and Laskar 2010), is a precise approach for a dynamical system when the inner body has a small mass in comparison with the other two bodies of the system (the central and the external/outer ones). This restricted model is no longer justified when in the presence of systems containing higher values for the mass ratios between their three bodies, which requires non-restricted models. For instance, it is possible to mention applications in the investigation of triple stars and triple asteroid systems (see for instance Carvalho et al. 2016a,b; Naoz et al. 2011; Naoz 2016; Fang et al. 2011; Prado 2014; Araujo et al. 2015). Despite not being the model considered in the present study, it is worth to mention the opposite configuration, the one with a massless body orbiting a massive inner binary system and which is called the "outer" restricted problem (Ferrer and Osacar 1994; Farago and Laskar 2010; Boué and Laskar 2009; Naoz et al. 2017). This model can be used when modeling the dynamics of many interesting problems, such as a spacecraft orbiting a binary planetary or an asteroid system or even a planet orbiting a binary star system (Giuliatti Winter et al. 2013; Naoz 2016).

Indeed, third-body perturbations have been object of several studies for many decades. Kozai (1959) obtained analytical expressions for the principal secular and longperiod terms of the disturbing function due to the lunisolar gravitational attractions. Musen et al. (1965) expanded this work including new terms in the disturbing function. Blitzer (1959) estimated lunisolar disturbances due to secular terms using methods of classical mechanics. Using the equatorial elements of the Moon, Kaula (1962) obtained the general terms of the disturbing function for the case of the lunisolar perturbations. Giacaglia (1973) calculated the secular longand short-period terms of the disturbing body, expressed in closed forms to eliminate the dependence on the true anomalies of the perturbed and perturbing bodies. Hough (1981) published a study considering the effects of lunisolar perturbations on spacecraft orbits close to the critical inclinations with respect to the geopotential $\left(i=63.4^{\circ}\right.$ and $\left.i=116.6^{\circ}\right)$.

Later, Broucke (2003) developed a second-order theory based on double-average techniques, extended by Prado (2003) to the fourth order. Other work that is worth to be checked is by Scheeres et al. (2001), Domingos et al. (2008) and Liu et al. (2012). They have considered this issue in order to develop approximated expressions for the third-body potential in order to get models that can represent full models, which would require numerical integrations with a good level of accuracy. These more recent papers have usually taken advantage of averaging techniques to study the thirdbody perturbations. Averaging methods have some advantages over full methods. They are usually faster in terms of computer time and effort. A more important point is that they are much less sensitive to initial conditions of the fast variables, as the initial true anomalies $\left(f\right.$ and $\left.f_{J}\right)$ of the spacecraft and third perturbing body. This approximation is valid when large number of revolutions are made by the spacecraft. For instance, for a given semimajor axis of $a=2510.3 \mathrm{~km}$ (orbit altitude $\sim 100 \mathrm{~km}$ ), the spacecraft's orbital period around Callisto is around 2.59 hours, while Jupiter's orbital period around its moon is about 400.53 hours, i.e., near 154.64 times slower.

It is also important to cite the idea of developing lifetime maps to analyze models for the third-body perturbation, as done in Gomes and Domingos (2016) to investigate the models presented in Domingos et al. $(2008,2014)$. In this work, Gomes and Domingos (2016) analyzed orbits around the Moon. A similar idea is used in Carvalho et al. (2017) to study some characteristics of orbits around the planet Mercury.

Regarding applications in modern space exploration, a system of planetary moons that has one of the largest number of celestial bodies with potential to be visited by space missions is the system of Jupiter, which has the Galilean moons, among others. A proposed mission that could visit some of its moons requires orbits having low altitude and high inclination to better map their gravity fields and surfaces. Recently, projects from the National Aeronautics Space Administration (NASA) and the European Space Agency (ESA) have been under development (NASA 2017; ESA 2014). The scientific objectives proposed for Europa and Ganymede, extended to Callisto, are: 1) characterization of the ocean layers and detection of subsurface water reservoirs; 2) topographical and geological analysis of the surface; 3) study of the physical properties of the icy crusts; 4) characterization of the internal mass distribution; 5) dynamics and evolution of the interiors; 6) investigation of Ganymede's tenuous atmosphere; 7) study of Ganymede's intrinsic magnetic field and its interactions with the Jovian magnetosphere. The three moons are believed to contain internal liquid water oceans, and therefore they can be considered central to understanding the habitability or not of icy worlds.

In this context, the present paper proposes to use a model that considers the eccentricity of the disturbing body, which can be used to improve the results available in the literature by means of investigating the role of the eccentricity of the disturbing body in the lifetime of low-altitude orbits around planetary moons. Different values (real and hypothetical ones) for the eccentricity of Jupiter are adopted in order to study in more detail the importance of this parameter. It means that hypothetical systems will be created, using the same physical parameters as from the JupiterCallisto system, but with hypothetical values for the eccentricity. It is also important to remember that the classical Keplerian/elliptical orbits are also disturbed by effects coming from the fact that the celestial bodies are not perfect 
spheres and that they do not have homogeneous distributions of mass. In order to improve the analysis, the effects due to the terms $J_{2}$ and $C_{22}$ of the non-uniform gravitational potential of Callisto are also considered.

The basic structure of this paper is shown next. In Sect. 1 an introduction to this subject is made; in Sect. 2 the equations of motion are obtained; in Sect. 3 the results of the numerical simulations are described and analyzed, and, finally, in Sect. 4, the conclusions are presented.

\section{Equations of motion and disturbing potentials}

The motion of a spacecraft under the influence of the forces coming from gravitational potentials can be described by the Lagrange planetary equations (Kovalevsky 1967), as a function of the semimajor axis (" $a$ "), eccentricity (" $e$ "), inclination (" $i$ ), argument of the periapsis (" $g$ "), longitude of the ascending node (" $h$ "), mean anomaly (" $\ell ")$, mean motion (" $n$ ") and the disturbing potential $R$.

In the present study, this potential is considered as composed of three terms that have the main roles in disturbing the orbital motion of a celestial body: $R_{2}$, which is the perturbation due to a third body present in the system we study, as the case of a spacecraft orbiting the moon and that receives a perturbation from the Earth and the Sun; $R_{J_{2}}$, which is the term that represents the flattening at the poles of the central body of the system; $R_{C_{22}}$, which is the term that denotes the elliptical shape of the equator of the central body.

It is necessary to obtain these equations of motion for the present study, so one is required to obtain analytical expressions for the disturbing forces.

\subsection{Double-averaged third-body perturbation}

The first force considered is the one responsible for the disturbances caused by a third body in an inclined and eccentric orbit. The spacecraft (mass $m$ ) is considered to be orbiting a moon (mass $m_{0}$ ), which is assumed to be around a planet, with a mass $m_{J}$. The mean motion $n$ appears in Kepler's third law: $G\left(m_{0}+m_{J}\right)=\mu_{J}=n_{J}^{2} a_{J}^{3}$, where $G$ is the gravitational constant assumed to be equal to $6.67259 \times 10^{-11} \mathrm{~kg}^{-1} \mathrm{~m}^{3} \mathrm{~s}^{-2}$. It is assumed that the disturbing body (planet) is in an elliptical and inclined orbit around the central body (moon) and its orbital elements are denoted by the subscript ' $J$ ': $a_{J}, e_{J}, g_{J}, h_{J}, i_{J}$ and $f_{J}$. The disturbing function due to a third body is given by (Murray and Dermott 2000)

$R=\frac{G\left(m_{0}+m_{J}\right)}{\left|\vec{r}_{J}-\vec{r}\right|}=\frac{\mu_{J}}{r_{J}} \sum_{k=0}^{\infty}\left(\frac{r}{r_{J}}\right)^{k} P_{k}(\cos (S))$

where $r$ and $r_{J}$ are the spacecraft and the disturbing body orbital distances from the central body, respectively.
Then a simplified dynamical system is obtained by expanding this disturbing potential up to second order $(k=2)$ in the Legendre polynomials $P_{k}$. It is possible to eliminate the fast angles of the problems (short-period terms), e.g. the mean anomalies of the bodies to get a simplified doubleaveraged model with potential to provide qualitative analysis of the system. This system avoids sensibilities in initial conditions for the orbital position of the two orbiting bodies, along with the significant gain of computer processing time. The disturbing function of the problem is averaged independently over the mean anomalies of the spacecraft and the third body. It is a good model when the spacecraft makes a large number of revolutions. The standard definition for the average used in this work is

$\langle F\rangle=\frac{1}{2 \pi} \int_{0}^{2 \pi}(F) d \ell$.

After averaging over the mean anomalies of the spacecraft $(\ell)$ and the third body $\left(\ell_{J}\right)$, the disturbing potential takes the following form:

$$
\left\langle\left\langle R_{2}\right\rangle\right\rangle=\frac{1}{4 \pi^{2}} \int_{0}^{2 \pi} \int_{0}^{2 \pi}\left(R_{2}\right) d \ell d \ell_{J}
$$

then it is possible to get the following model (Tresaco et al. 2016; Carvalho 2016):

$$
\begin{aligned}
\left\langle\left\langle R_{2}\right\rangle\right\rangle= & -\frac{15}{16} n_{J}^{2} a_{J}^{2}(A+B+C+D+E+F \\
& +G+H)
\end{aligned}
$$

with

$A=\frac{1}{4} e^{2}(\cos (i)-1)^{2}\left(\cos ^{2}\left(i_{J}\right)-1\right) \cos \left(2 g-2 h+2 h_{J}\right)$,

$B=\frac{1}{4} e^{2}(\cos (i)-1)^{2}\left(\cos ^{2}\left(i_{J}\right)-1\right) \cos \left(2 g+2 h-2 h_{J}\right)$,

$C=\frac{e^{2}}{2} \sin (i) \sin \left(i_{J}\right)(\cos (i)-1) \cos \left(2 g-h+h_{J}\right)$,

$D=\frac{e^{2}}{2} \sin (i) \sin \left(i_{J}\right)(\cos (i)+1) \cos \left(2 g+h-h_{J}\right)$

$E=-\frac{3}{10}\left(e^{2}+\frac{2}{3}\right)\left(\cos ^{2}(i)-1\right)\left(\cos ^{2}\left(i_{J}\right)-1\right)$ $\times \cos \left(2 h-2 h_{J}\right)$,

$F=-\frac{3}{10}\left(e^{2}+\frac{2}{3}\right) \sin (2 i) \sin \left(2 i_{J}\right) \cos \left(2 h-2 h_{J}\right)$,

$G=\frac{1}{2} e^{2}\left[\left(\cos ^{2}(i)-1\right)\left(\cos ^{2}\left(i_{J}\right)+1\right)\right.$

$$
\left.+2 \sin ^{2}(i) \sin ^{2}\left(i_{J}\right)\right] \cos (2 g),
$$

$H=-\frac{3}{10}\left(e^{2}+\frac{2}{3}\right)\left[\left(\cos ^{2}\left(i_{J}\right)+1\right) \cos ^{2}(i)\right.$

$$
\left.+\left(\cos ^{2}\left(i_{J}\right)-\frac{5}{3}\right)+2 \sin ^{2}(i) \sin ^{2}\left(i_{J}\right)\right],
$$


where the subscript $J$ denotes variables referred to the disturbing body (Jupiter in our numerical experiments) and $n_{J}$ is the mean motion of this body. The original disturbing functions are given in terms of the true anomalies. To compute the double integral in the averaging process, which is presented in terms of mean anomalies, the following wellknown relations were used (see Brouwer and Clemence 1961):

$\sin (f)=\frac{\sqrt{1-e^{2}} \sin (E)}{1-e \cos (E)}$,

$\cos (f)=\frac{\cos (E)-e}{1-e \cos (E)}$,

$r=a(1-e \cos (E))$,

$d \ell=(1-e \cos (E)) d E$,

with $E$ denoting the eccentric anomaly of the spacecraft. Analogous orbital parameters carrying the subscript $J$ represent parameters referred to the third body.

\subsection{Perturbations due to central body non-uniform shape}

Since the orbits required for scientific missions around a planetary moon are desired to be low altitude $(\sim 100 \mathrm{~km})$ ones and also near-circular and near-polar, the present paper concentrates in this type of orbits. It means that the present study needs to take into account the perturbation due to the non-uniform distribution of mass of the central body. The effects due to planetary moon flattening at the poles $\left(J_{2}\right)$ and due to the elliptic shape of its equator $\left(C_{22}\right)$ are included, as addressed in previous work (Scheeres et al. 2001; Paskowitz and Scheeres 2006; Lara and Russell 2006; Carvalho et al. 2012b,a; Vilhena De Moraes et al. 2016).

Considering the equatorial plane of the central body (here a planetary moon) as the reference plane, the disturbing potential related to its distribution of mass can be written in the form

$$
\begin{aligned}
U_{M}= & -\frac{\mu}{r}\left[\sum_{n=2}^{6}\left(\frac{R_{p}}{r}\right)^{n} J_{n} P_{n}(\sin (\phi))\right. \\
& \left.-\left(\frac{R_{B}}{r}\right)^{2} C_{22} P_{22}(\sin (\phi)) \cos (2 \lambda)\right]
\end{aligned}
$$

where $\mu$ is the gravitational constant of the body, $R_{B}$ is the equatorial radius of the body, $P_{n}$ are the Legendre polynomials, $P_{n m}$ the associated Legendre polynomials, the angle $\phi$ is the latitude of the orbit with respect to the equator of the body, the angle $\lambda$ is the longitude measured from the direction of the longest axis of the planet. Using spherical trigonometry, it is also possible to show that $\sin (\phi)=$ $\sin (i) \sin (f+g)$.
The Legendre polynomial for the zonal term $J_{2}$ and the Legendre associated function for the sectoral term $C_{22}$ can be written in the form (Giacaglia et al. 1970)

$$
\begin{aligned}
& P_{2}(\sin (\phi))=\frac{1}{2}\left(3 s^{2} \sin ^{2}(f+g)-1\right), \\
& P_{22}(\sin (\phi)) \cos (2 \lambda) \\
& =6\left(\xi^{2} \cos ^{2}(f)+\chi^{2} \sin ^{2}(f)+\xi \chi \sin (2 f)\right) \\
& \quad-3\left(1-s^{2} \sin ^{2}(f+g)\right),
\end{aligned}
$$

with the shortcuts $\xi=\cos (g) \cos (h)-c \sin (g) \sin (h), \chi=$ $-\sin (g) \cos (h)-c \cos (g) \sin (h), s=\sin (i)$ and $c=\cos (i)$.

Now, it is possible to write the potential given in Eq. (17) as a function of the orbital elements. For this purpose, one can use Eq. (18) for the zonal term $J_{2}$ and the relation $\mu=$ $n^{2} a^{3}$, such that

$U_{20}=-\frac{1}{2} \frac{a^{3}}{r^{3}} \epsilon n^{2}\left(3 s^{2} \sin ^{2}(f+g)-1\right)$.

Analogously, it is possible to take Eq. (19) for the sectoral perturbation (Giacaglia et al. 1970). Thus,

$$
\begin{aligned}
U_{22}= & \frac{a^{3}}{r^{3}} \delta n^{2}\left(6 \xi^{2} \cos ^{2}(f)+6 \chi^{2}(\sin (f))^{2}\right. \\
& \left.+12 \xi \chi \sin (2 f)-3+3 s^{2} \sin ^{2}(f+g)\right)
\end{aligned}
$$

where $\delta=C_{22} R_{B}^{2}$.

Since $f$ is the true anomaly of the artificial satellite, one can make the choice of working with a long-period dynamical model, as done for the third-body potential. It requires averaged disturbing potentials, so the concept of averaging method (see Eq. (2)) is again applied for the models presented in Eqs. (20) and (21) to obtain a single-averaged model where the short-period terms are eliminated. This fact accelerates the integration of the equations of motion and provides a qualitative analysis of the system by avoiding sensibility related to the initial conditions. The development of the equations is carried out in closed form to avoid expansions in eccentricity and inclination. For this purpose, it is necessary to perform algebraic manipulations where known equations from celestial mechanics are used, namely Eqs. (22) and (23),

$\frac{a}{r}=\frac{(1+e \cos (f))}{\left(1-e^{2}\right)}$,

$d l=\frac{1}{\sqrt{1-e^{2}}} \frac{r^{2}}{a^{2}} d f$.

After applying the averaging process in Eq. (20) and after some algebraic manipulations, the averaged disturbing potential due to the flattening at the poles becomes

$$
\left\langle R_{J_{2}}\right\rangle=-\frac{1}{4} \frac{\epsilon n^{2}}{\left(1-e^{2}\right)^{3 / 2}}\left(3 \sin ^{2}(i)-2\right)
$$


where $n$ is the mean motion of the spacecraft (see also Cardoso dos Santos et al. (2015)). The parameter $\epsilon$ is given by

$\epsilon=J_{2} R_{B}^{2}$.

Now, using Eq. (21), the variables $\xi$ and $\chi$ to develop the potential due to the equatorial ellipticity of the central body, we get

$\left\langle R_{C_{22}}\right\rangle=-\frac{3}{2} \frac{\delta n^{2}}{\left(1-e^{2}\right)^{3 / 2}}\left(\cos ^{2}(i)-1\right) \cos (2 h)$.

The parameter $\delta$ is given by

$\delta=C_{22} R_{B}^{2}$.

In Eq. (26) the longitude of the ascending node $h$ is replaced by the expression $h=h_{0}-\omega t$ given by Meyer et al. (1994), Giacaglia (1973) and Sehnal (1960), where $t$ is the time and $\omega$ is the body's rotation speed. This expression $h=h_{0}-\omega t$ (see Fig. A1 in Meyer et al. 1994) relates the longitude of the ascending node of the orbit with respect to Callisto $(h)$ to the inertial longitude of the ascending node $\left(h_{0}\right)$.

\subsection{Equations of motion}

After obtaining the potentials for modeling the physical system of our interest, the total disturbing potential $R=$ $\left\langle\left\langle R_{2}\right\rangle\right\rangle+\left\langle R_{J_{2}}\right\rangle+\left\langle R_{C_{22}}\right\rangle$ is replaced in the classical Lagrange planetary equations (Kovalevsky 1967) in order to integrate the motion of the spacecraft under these gravitational effects. Note that in the second averaging process the orbital elements of the spacecraft are constant, so that $\left\langle R_{J_{2}}\right\rangle+\left\langle R_{C_{22}}\right\rangle=\left\langle\left\langle R_{J_{2}}\right\rangle\right\rangle+\left\langle\left\langle R_{C_{22}}\right\rangle\right\rangle$ and, thefore $R=\left\langle\left\langle R_{2}\right\rangle\right\rangle+\left\langle\left\langle R_{J_{2}}\right\rangle\right\rangle+\left\langle\left\langle R_{C_{22}}\right\rangle\right\rangle$.

Those equations are shown below

$$
\begin{aligned}
\frac{d a}{d t} & =\frac{2}{n a} \frac{\partial R}{\partial \ell}, \\
\frac{d e}{d t} & =\frac{-\sqrt{1-e^{2}}}{n a^{2} e} \frac{\partial R}{\partial g}+\frac{1-e^{2}}{n a^{2} e} \frac{\partial R}{\partial \ell}, \\
\frac{d i}{d t} & =\frac{1}{n a^{2} \sqrt{1-e^{2}} \operatorname{sen}(i)}\left(\frac{\partial R}{\partial g} \cos (i)-\frac{\partial R}{\partial h}\right), \\
\frac{d h}{d t} & =\frac{1}{n a^{2} \sqrt{1-e^{2}} \operatorname{sen}(i)} \frac{\partial R}{\partial i}, \\
\frac{d g}{d t} & =\frac{\sqrt{1-e^{2}}}{n a^{2} e} \frac{\partial R}{\partial e}-\frac{\cos (i)}{n a^{2} \sqrt{1-e^{2}} \operatorname{sen}(i)} \frac{\partial R}{\partial i} .
\end{aligned}
$$

\section{Results and discussions}

The present paper intends to analyze the magnitude of the effects of the third body's eccentricity over a massless spacecraft orbiting a central body. The effects of $J_{2}$ and $C_{22}$ are considered in this analysis and different values for the orbital eccentricity of the third body are assumed, such that the results are able to provide some insights about the dynamics of a spacecraft around a body with elliptical shape orbiting a massive body. The Jupiter-Callisto system is used in the present study and the ideas developed here can be extended to other systems, such as moons around massive planets, asteroids (NEAs) and comets with significant close approaches with respect to the Sun. The purpose of the present investigation is attained by making lifetime maps for different values of the eccentricity of the disturbing body.

This procedure helps the search for low-altitude nearpolar natural orbits around Callisto (as well for other bodies) that survive for long periods of time, which we assume in the present work as lifetimes longer than one year. This search for orbits with longer lifetimes is made by mapping different initial conditions for the orbital elements due to a spacecraft orbiting the moon. Such orbits are affected by disturbances due to the non-sphericity of the central body and especially by the Kozai-Lidov mechanism (Kozai 1962; Lidov 1962) coming from the third body (here assumed to be Jupiter). This is a well-known effect, which allows a body to periodically exchange its inclination with eccentricity, which creates a significant decrease in the lifetimes to values of tens or a few hundreds of days (Prado 2003) for the case of lowaltitude orbits $(\sim 100 \mathrm{~km})$. Although the effect coming from the third body makes the eccentricity to grow, the disturbing effects coming from the irregular shape of the central body $\left(J_{2}\right.$ and $C_{22}$ ) tends to counterbalance the third-body disturbances at some level. However, the term $C_{22}$ sometimes can contribute with the third body's effect by counterbalancing the effect of $J_{2}$ (see Fig. 5). The final impact on the dynamics presents some increase in the orbit lifetimes in the presence of significant values for these higher gravitational inhomogeneities, especially considering $J_{2}$. Besides, as seen in previous work (see Carvalho et al. (2010) and the references therein), the presence of coupling effects due to the $C_{22}$ perturbation may imply in lowering the periapsis altitude due to the periodic oscillations produced in the eccentricity. In order to provide useful information for missionplanning purposes, it is necessary to incorporate the more relevant disturbing effects in the dynamical model, which in the present investigation justifies the presence of effects due to $J_{2}$ and $C_{22}$, with their high- and same-order values.

Previous work (Scheeres et al. 2001; Broucke 2003; Prado 2003) usually considered a circular planar doubleaveraged model for the third-body perturbation, which is a good approximation for the Galilean moons and other moons of the Solar System. However, it is possible to have some insights about the dynamics under the effects of a disturbing body in eccentric orbit by means of creating hypothetical systems with different values for its orbital eccentricity $\left(e_{J}\right)$, in order to measure how strong is the impact 
of this parameter in the lifetimes of the orbits of a spacecraft around the central body, assumed to have an ellipsoidal shape. Therefore, selecting hypothetical different eccentricities for Jupiter's orbit, and generating these lifetime maps for a spacecraft orbiting Callisto considering these different scenarios, is also an important opportunity to test the present double-averaged model that considers the eccentricity and the inclination of the disturbing body. Due to the purpose of having a model which can also be useful for orbiters around NEAS and comets, especially having in mind these last ones as objects with rather eccentric orbits under the massive Sun's gravitational field, the investigation of the role of disturber's inclination $\left(i_{J}\right)$ is left for a next communication where other discussions including critical inclination and other important issues can be more properly treated and also with applications to different celestial bodies. As Callisto's motion around Jupiter is almost planar, and the same happens with the majority of the moons with higher interest for future space exploration, the discussion as regards the role of the disturber's inclination by mean of generating lifetime maps is, presently, delayed.

In order to test this model for the third-body perturbation, it was integrated and mapped orbits obtained under different initial conditions for the inclination $(i)$, varying between $30^{\circ}$ and $150^{\circ}$, and semimajor axis (a) varying between $2460.3 \mathrm{~km}$ and $2510.3 \mathrm{~km}$, which corresponds, for a Callisto radius of $2410.3 \mathrm{~km}$, to averaged altitudes of $50 \mathrm{~km}$ and $100 \mathrm{~km}$, respectively. The initial conditions for the other orbital elements of the spacecraft orbit are $e=0.01, g=270^{\circ}$ and $h=90^{\circ}$.

The values for the gravity parameters $\left(J_{2}=3.27 \times\right.$ $10^{-5}$ and $\left.C_{22}=1.02 \times 10^{-5}\right)$ are taken from Anderson et al. (2001). The orbital parameters for the JupiterCallisto system are (Source: http://ssd.jpl.nasa.gov/): $a_{p}=$ $1,882,700 \mathrm{~km}, e_{p}=0.0074, g_{p}=52.643^{\circ}, h_{p}=63.552^{\circ}$, $i_{p}=0.192^{\circ}$ and $n_{p}=21.5710728^{\circ} /$ day. The value for the mean radius of Callisto $R_{B}=2,410.3 \mathrm{~km}$ is taken from the same source.

A first test of lifetime maps considering the models presently introduced can be seen in Fig. 1, where the value chosen for the orbital eccentricity of the spacecraft does not impact the lifetime as significantly as the choice of a value of the inclination when it is close to a polar orbit $\left(i=90^{\circ}\right)$. The range available for the spacecraft's orbital eccentricity is small due to the fact that the spacecraft is only about $100 \mathrm{~km}$ of altitude (with critical eccentricity $e_{\max } \sim 0.0398$ ), which makes the values of the lifetime very sensible in such dynamics. In this scenario, it creates fast collisions with the surface of Callisto under small increases of the perturbations over the orbital eccentricity. Therefore, Fig. 1 presents results for a lifetime map as a function of initial eccentricity and initial inclination. It is possible to note in the results the expected effect of the lifetimes decreasing with the increasing of the value of the initial eccentricity. The Kozai-Lidov effect is present in decreasing the lifetime of orbits closer to $90^{\circ}$ of inclination (darker colors are close to this angle). Moreover, it is in order is to explain that the orbits do not survive for initial eccentricities higher than $e=0.039$ due to the proximity of the spacecraft with Callisto and due to the strong perturbations coming to the third body that increase the value of the eccentricity, reaching the small value of 0.039 in a short time, which corresponds to a distance of the periapsis equal to the mean radius of Callisto.

The conservation of $H_{z}=\sqrt{1-e^{2}} \cos (i)$, i.e., the component of the spacecraft's orbital angular momentum, which is parallel to Jupiter's angular momentum, is shown in Fig. 2. Indeed, under the regime of this restricted dynamical model, the conservation of this quantity allows the connection of the two parameters, orbital eccentricity and inclination, as being "exchanged" or "traded", one parameter by the other. Therefore, for spacecrafts with orbits which are near-circular and highly inclined, it is possible to occur that they become very eccentric. Thus, this growth of the eccentricity allows: 1) Impact with other bodies of the system; 2) Eccentricity increasing until the orbit reaches enough energy to escape from the system. For the more general case, where the spacecraft is replaced by natural celestial bodies orbiting others under a third-body perturbation, it is possible to occur the destruction of this celestial body due to the tidal forces, once the increasing eccentricity will make the celestial body's periapsis to pass/cross inside the Roche limit related to the more massive central body. Another important issue to mention is that the exchange of eccentricity and inclination creates oscillations which generates impacts in lower-altitude orbits, since they are closer to the central body's surface. These oscillations can be seen in Fig. 3. The plots presented in this figure are in accordance with another expected effect due to the Kozai-Lidov mechanism, which is to cause a libration of the argument of the periapsis $g$ around angles $g=90^{\circ}$ or $g=270^{\circ}$. Figure 4 presents the components of the eccentricity vector integrated for a period of 100 years. In fact, the plots in Fig. 3 help to interpret the results in Fig. 4, with high-inclination orbits $\left(i\right.$ between $45^{\circ}$ and $135^{\circ}$ ) presenting a libration of the argument of periapsis in the approximate interval of $g=243^{\circ}$ and $g=297^{\circ}$. This behavior is a result of the Kozai-Lidov mechanism, which makes $g$ librate when the orbits are highly inclined. Orbits with low-inclination present the argument of periapsis circulating, which explain the circles and ellipses covering the full range of $360^{\circ}$ in the second and third plots in Fig. 4.

The following test was made for models considering different perturbing forces on the disturbing potential: (1) $R_{2}$; (2) $R_{2}+J_{2}$; (3) $R_{2}+J_{2}+C_{22}$. Figure 5 presents the results for lifetime maps considering these three scenarios. From scenario (1), with perturbation coming from the third body, until scenario (3) with the sum of the effects due to the non-uniform distribution of mass of Callisto. The result 
for the scenario (2) present a map that is similar to those referred to cases (1) and (3); however, with some significant differences in the range of the inclination that produce longer lifetimes. As expected, $J_{2}$ acts as a natural protecting mechanism by smoothing the disturbing effects coming from the third body. This test was made considering the real value of the eccentricity for the Jupiter-Callisto system $\left(e_{p}=0.0074\right)$. The results show the third-body disturbance as the main perturbation in this system, especially due to the mass of the disturbing body Jupiter and the smaller magnitude of the effects due to the gravity terms $J_{2}$ and $C_{22}$ of Callisto. The presence of extra perturbations in the system, as the case of terms like $J_{2}$, have a role in precessing the argument of the periapsis and the longitude of the ascending node of the spacecraft's orbit. However, it does not affect the lifetimes of the orbit as in this double-averaged model, since they are a function of the orbital eccentricity. The gravity term $C_{22}$ also acts as a protecting mechanism once it is coupled with other orbital perturbations. As $C_{22}$ is one of the perturbations considered in our dynamics, Figs. 6 and 7 are tests created to map lifetimes varying the initial value of the longitude of the ascending node $h_{0}$. In addition, intervals with values for argument of periapsis $\left(g_{0}\right)$, inclination $\left(i_{0}\right)$ and eccentricity $\left(e_{0}\right)$ are mapped. It clearly shows values for this orbital parameter that can increase the lifetimes of the orbits. We see, for example, that $h_{0}=90^{\circ}$ is able to produce a small increase in the polar orbits lifetime, e.g., $i_{0}=90^{\circ}$. In fact, the choice of $h_{0}=90^{\circ}$ in the present numerical simulations was made based on this result. Therefore, convenient choices for the angles of the problem, as noted for the inclination and for the argument of periapsis of a spacecraft orbiting Callisto, are very important regarding extending the lifetimes of a space mission that intends to orbit this body.

Once orbits with high inclination are desired for space mission applications and the effect due to $C_{22}$ is coupled in our dynamics, Fig. 7 shows tests made by creating lifetime maps considering variations in the initial value of the longitude of the ascending node $h_{0}$ and inclination $i_{0}$. This figure clearly shows, concerning the initial values for $h_{0}$, that this parameter can increase the lifetimes of the orbits. For instance, $h_{0}=90^{\circ}$ is able to produce a small increase in a polar orbit. In fact, the choice for $h_{0}=90^{\circ}$ in the present numerical simulations was made based on this result. Therefore, convenient choices for the angles of the problem of a spacecraft orbiting Callisto are very important regarding extending the lifetime of a space mission that intends to orbit this body. The first plot presented in Fig. 6 justifies the choice for $h_{0}=90^{\circ}$ as the value that produces higher values of lifetimes in high-inclination orbits, the orbits of main interest for the present investigation. The second plot in the same figure shows values with respect to the relation $g_{0}=k \pi$ radians (with $k$ an integer) as better choices to increase the lifetime when one considers polar orbits. These differences are highlighted in Fig. 7, where it is presented lifetime maps as a function of the initial inclination $i_{0}$ and initial longitude of the node of the spacecraft's orbit $h_{0}$. It also considers the cases for $g_{0}=270^{\circ}$ and $180^{\circ}$. This result appears similar to the ones presented in Condoleo et al. (2016) considering the dynamics of a spacecraft orbiting Callisto under the disturbing potentials $R_{2}$ and $J_{2}$.

Here, Figs. 8 to 10 represent some orbits integrated for longer periods of time to provide some information as regards the long-term dynamics. The results in these figures are similar to those presented in Figs. 1, 2 and 4 of Prado (2003). But these considered a Lunar orbiter. Figure 8 confirms the results presented in Fig. 1, where the choice of different initial values for the eccentricity does not impact the evolution of the inclination. Similar results appear when other values for inclination are considered and for this reason, they are omitted here. It is worth to mention that the result presented in Fig. 9, where it is possible to verify that the more circular the initial orbit is, the longer are the lifetimes presented. Figures 8 to 10 present the expected exchange of eccentricity and inclination, which corresponds to the previously mentioned conservation of the z-component of the orbital angular momentum $H_{z}=\sqrt{1-e^{2}} \cos (i)$ (see Fig. 2). As observed in the amplitude of the oscillations presented in these figures, the small changes in one parameter will impact on the existence of small changes in the other as well.

Next, the effects of the eccentricity are measured. To perform this task, all the parameters of the Jupiter-Callisto system are kept constant, except for the eccentricity of the disturbing body, which is Jupiter for this problem. It means that hypothetical systems are used to measure the effects of the eccentricity, without varying the other parameters, such that it is possible to be sure that all the modifications in the results are coming from the eccentricity of the primaries. Figures 11 and 12 show a graphical general view of the lifetimes. It also contribute to evaluate how significant is this protecting mechanism due to the gravity terms ( $J_{2}$ and $C_{22}$ ), especially in the presence of a very massive third body (as the case of Jupiter). We choose different values for the eccentricity of the disturbing body $e_{p}=$ $\{0.1 ; 0.2 ; 0.3 ; 04 ; 0.5$ and 0.6$\}$ in order to create JupiterCallisto like systems which are able to show how much the harmonics are able to help on increasing lifetimes under extreme third-body perturbations. These results are presented in Figs. 11 and 12. It is worth of note the lifetime maps presented in Fig. 11 very similar to the ones shown in Fig. 5. It indicates that the significant magnitude of the gravity terms $J_{2}$ and $C_{22}\left(\sim 10^{-5}\right)$, is strong enough to naturally protect the orbit against an increase of the lifetime with a very massive disturbing body in a significantly eccentric orbit. Due to the fact that more eccentric orbits make the spacecraft to pass closer to the disturbing body, and vice 
versa, it is expected an increase of the disturbing effects due to its gravitational attraction. Naturally, as observed in the lifetime maps presented in Figs. 11 to 12, when the eccentricity $e_{J}$ increases, the disturbing effects become stronger and the lifetime of the orbits tend to decrease.

A first look at the results confirms the presence of the expected Kozai-Lidov effects in reducing the lifetime of orbits with high values for the inclination. These results are compatible with the ones shown in Prado (2003), which performed a similar study for the particular value for the semimajor axis of four radius of the moon considering only the term $R_{2}$. It is a high value of altitude for the orbit in comparison with the ones presented in the present study, which are in a range of values between $50 \mathrm{~km}$ and $100 \mathrm{~km}$. Looking at the results obtained using the real value for the eccentricity of Callisto (Fig. 5), it is noted that orbits with initial semimajor axis $a_{0}$ above $2480 \mathrm{~km}$ usually present lifetimes of about 300 days or more. Below this value, the lifetime depends more significantly on the inclination of the orbit. Figure 5 shows a general view of these lifetimes. In general, for the cases with orbital inclination between $\sim 42^{\circ}$ and $\sim 137^{\circ}$, there are collisions before the time reached this limit. Those results are very important when planning missions to minor bodies of the Solar System (planetary moons, asteroids, comets) under the massive third-body perturbation of the planets or Sun, since polar orbits are good candidates for surface observations, mapping and characterization. In order to attain these scientific goals, high-inclination orbits offer the best coverage. The results show the eccentricity of the primaries impacting in reducing the lifetimes of orbits, in particular in the ones closer to the moon. The physical interpretation is not difficult. Larger eccentricities of the primaries allow shorter distances for the perturbing body, so the effects are larger when the moon is at the periapsis. Of course the planet is far way during the passage of the moon near the apoapsis, which reduces the perturbation. So, an analysis is required in order study the balance of those effects, and the results show that there is an expected increase in the perturbation with the eccentricity $e_{p}$, reducing the lifetimes of the orbits. The reductions are not significant for $e_{p}=0.1$ due to the protecting mechanism of $J_{2}$ and $C_{22}$, and also due to the fact that Callisto is the more distant object among the Galilean moons. This is not a problem in this analysis, because new reductions are noted in those minimum lifetimes when the eccentricity of the primaries is 0.2 , especially for $a_{0}$ above $2500 \mathrm{~km}$ in the second plot of Fig. 11. For values of $e_{p}=0.4$ and above, the plots are very different, with blue color dominating the results. It means that much smaller lifetimes (Fig. 12). These results are expected, but a quantification is important, as done in the present paper.

Indeed, a more rigorous exploration of these orbital parameters can be made using some values studied in Con- doleo et al. (2016), where the authors explore some conditions for freezing orbits around planetary satellites with a disturbing potential taking into account the effects of the third body and of the flattening at the poles $\left(J_{2}\right)$. Despite the present study taking into account a more general model with the presence of $C_{22}$, the values presented in the work of Condoleo et al. can be used as good initial guide on finding initial conditions which produce longer lifetimes, despite the existence of some extra disturbing potentials. For instance, one of the conditions that the authors find to freeze a polar orbit is to set $g_{0}=0^{\circ}$ or $g_{0}=180^{\circ}$, i.e., these values freeze polar orbits when the potential $R_{2}+J_{2}$ is taken into account. This is the same result found in Figs. 6 and 7, now with the additional effects due to $C_{22}$. Therefore, some numerical explorations can be made by reproducing some previous results considering $g_{0}=180^{\circ}$ instead of $g_{0}=270^{\circ}$. An evidence of this effect of selecting this angle to increase the lifetime of orbits can be observed by analyzing Figs. 13 to 14. In these cases $g_{0}=180^{\circ}$ is able to protect significantly the surviving time of the polar orbits, despite the increase of the disturbance coming from the third body (Jupiter). It is highlighted when a comparison of the results shown in Figs. 13 and 14 is made with the results shown in Figs. 11 and 12 . The lifetime map $i_{0}$ vs. $a_{0}$ considering $e_{J}=0.01$ is omitted in Fig. 13 due to similarity with the lifetime map for the real value $e_{J}=0.0074$ (first plot in Fig. 13), as happened with the results for $g_{0}=270^{\circ}$.

Figures 15 and 16 present the components of the eccentricity vector considering the disturbing potential $R_{2}+J_{2}+$ $C_{22}$. Different scenarios $\left(g_{0}=270^{\circ}\right.$ and $\left.g_{0}=180^{\circ}\right)$ are assumed and similar analysis can be done, as presented in Fig. 4. It is also important to highlight such figures as useful plots to identify the existence of libration and circulation motions.

Finally, Figs. 17 and 18 show the orbit time as a function of the inclination, for different values of initial semimajor axis $a_{0}$ and initial eccentricity $e_{0}$. The results considering again scenarios with $g_{0}=270^{\circ}$ and $g_{0}=180^{\circ}$ confirm the relevance of the present discussion, especially regarding an appropriate choice of the orbital parameters capable to extend the lifetimes of orbits. In all the cases, despite the selection of different initial values for semimajor axis $a_{0}$ and eccentricity $e_{0}$, the choice for $g_{0}=180^{\circ}$ was able to extend from tens up to hundreds of days the lifetimes of the orbits. Considering the scenario for an Europa orbiter with a dynamical model under the disturbances of the averaged models for $R_{2}$ and $J_{2}$, one can check similar plots in Figs. 8 and 9 of Scheeres et al. (2001). In this work, the authors choose to vary the initial argument of periapsis $g_{0}$ instead of varying the initial semimajor axis $a_{0}$ or the initial eccentricity $e_{0}$ as an alternative method to find higher values to extend the lifetime of a Galilean moon orbiter. 


\section{Conclusions}

In the context of the inner restricted three-body problem, the present paper studied the lifetime of orbits for a spacecraft traveling around the moon Callisto, which is in an eccentric and almost planar orbit around Jupiter. This system is chosen as a model for the application of similar studies to other celestial bodies, especially minor bodies of the Solar System, which are potential candidates to receive a space mission in the future. One of the goals of the present study was to investigate the impact of the massive disturbing body's orbital eccentricity in the lifetime of a spacecraft's orbit around a body with an ellipsoidal shape. The mathematical model used here considers the effects of the third body in elliptical and inclined orbits and the irregular shape of the central body by using the terms $J_{2}$ and $C_{22}$.

For the simulations, the system Jupiter-Callisto is used to give the basic physical data of masses, distances and shapes of the bodies, but the eccentricity is set with different values to show the importance of this parameter on more disturbed dynamics, such as the case of comets that are in rather eccentric orbits around the Sun. During the investigation, values for some orbital elements were determined to increase the lifetime of orbits around Callisto. These results are especially important concerning high-inclination orbits, the desired ones for global mapping and characterization. However, as these orbits usually present shorter lifetimes due to the Kozai-Lidov mechanism, the present investigation has importance, because it helps to find the parameters which are able to extend these lifetimes, increasing the available time for space mission operations around these polar and quasipolar regions. The Kozai-Lidov effects clearly and strongly appear in the systems simulated, showing the importance of finding the combination of parameters $\left(g_{0}=180^{\circ}\right.$ and $h_{0}=90^{\circ}$ ) that was able to extend the lifetimes of the polar orbits to higher values. Considering orbits with inclinations above $90^{\circ}$, some symmetries (which are not perfect in some cases) are noted in the results around the polar orbit, which means that orbits with inclination of $90^{\circ}+x^{\circ}$ and $90^{\circ}-x^{\circ}$ have very similar lifetimes.

Regarding the effects of the irregular shape of the moon, they are not very large for this system. This is because Callisto has a near spherical shape, with $J_{2}=3.27 \times 10^{-5}$ and $C_{22}=1.02 \times 10^{-5}$. In a short-time orbit, the effects due to these gravity terms are compared to the model considering only the third-body perturbation. Hence, the effects will be much stronger for bodies with more irregular shapes.

As expected, when considering the effects of hypothetical higher values for the orbital eccentricity of the massive third body (Jupiter), the more eccentric the orbit, the larger impact this body will produce on decreasing the lifetime of the orbit. On the other hand, the more circular is the orbit, the less affected it will be by the third-body perturbation.
Therefore, the general contribution of the present paper is to show the lifetimes of orbits around moons in eccentric orbits, generating maps that can be used for mission designers. It confirms the lifetime maps as an efficient tool to map and find the initial conditions that extend the surviving time of the orbits, especially those highly disturbed and with high values of inclination. Of course, along with this tool of the lifetime maps, other techniques can be used. For instance, more details as regards the mathematical and physical aspects that lead to these dynamical behaviors might be discussed. These aspects can be explored in a future communication. Similar ideas can be extended to applications in planetary physics. A good example of an interesting system to be investigated using the present models and techniques is the system composed of the dwarf planet Haumea, two small moons and a recently discovered ring (Ortiz et al. 2017).

Acknowledgements The authors wish to honor God for all the opportunities given during the development of this work. Special thanks are extended to reviewers for their valuable ideas and suggestions. The authors also acknowledge the support from UNESP, UFRB, INPE, UNIFESP. This work was sponsored by the São Paulo Research Foundation-FAPESP (processes 2013/26652-4, 2012/125399, 2016/24561-0, 2016/14665-2, 2012/21023-6, 2011/05671-5, 2011/08171-3) and the National Council for Scientific and Technological Development-CNPq (contracts 406841/2016-0, 301338/2016-7, 306953/2014-5, 420674/2016-0).

\section{Appendix}

The present appendix shows the figures containing the results discussed in the text. The simulations and plots were made with proper computer codes developed using the softwares Maple and Gnuplot.

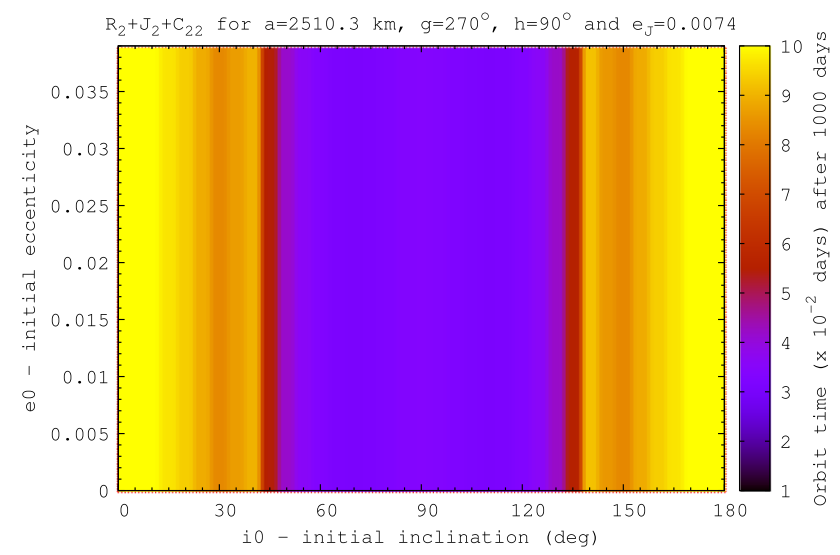

Fig. 1 Lifetime map ( $e_{0} \quad$ vs. $\left.i_{0}\right)$ considering the effects of $R_{2}+J_{2}+C_{22}$. Initial conditions: $a_{0}=2510.3 \mathrm{~km}, g_{0}=270^{\circ}$, $h_{0}=90^{\circ}$ and $e_{J}=0.0074$. The vertical bar on the right gives the value of the lifetimes in hundreds of days for an integration of 1000 days. Each unit corresponds to hundred days 


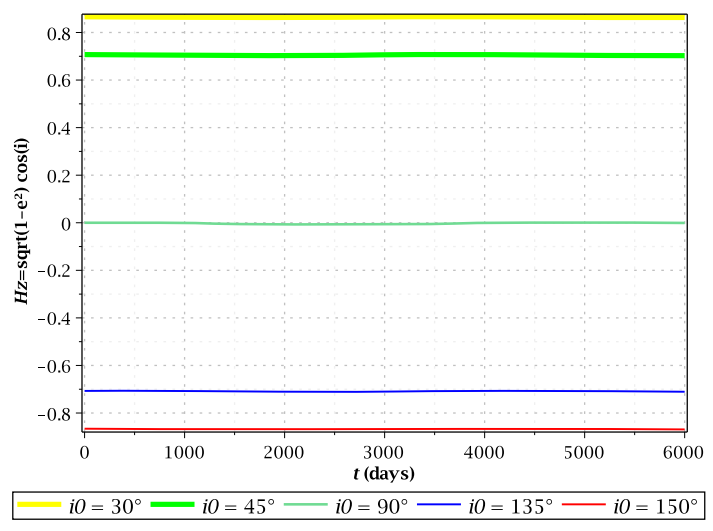

Fig. 2 Propagation of the $z$-component of the orbital angular momentum $H_{z}=\sqrt{1-e^{2}} \cos (i)$. Initial conditions: $a_{0}=2510.3 \mathrm{~km}$, $g_{0}=270^{\circ}, h_{0}=90^{\circ}$ and $e_{J}=0.0074$. The effects are shown that are due to $R_{2}$. Numerical propagation for 6000 days
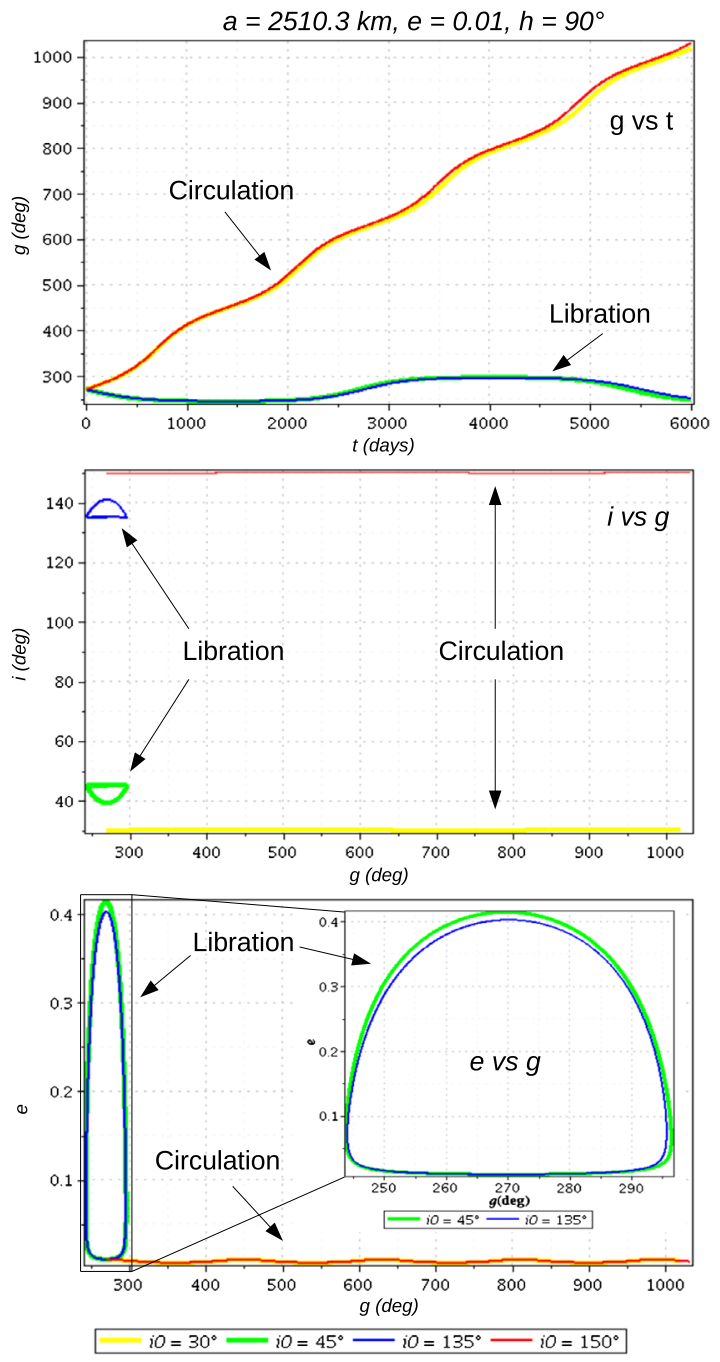

Fig. 3 Propagation of the eccentricity (e), inclination $(i)$ and argument of the periapsis $(g)$ considering different values for the initial inclination $i_{0}$. Initial conditions: $a_{0}=2510.3 \mathrm{~km}, e_{0}=0.01, g_{0}=270^{\circ}$, $h_{0}=90^{\circ}$ and $e_{J}=0.0074$. The effects are shown that are due to $R_{2}$. Numerical propagation for 6000 days

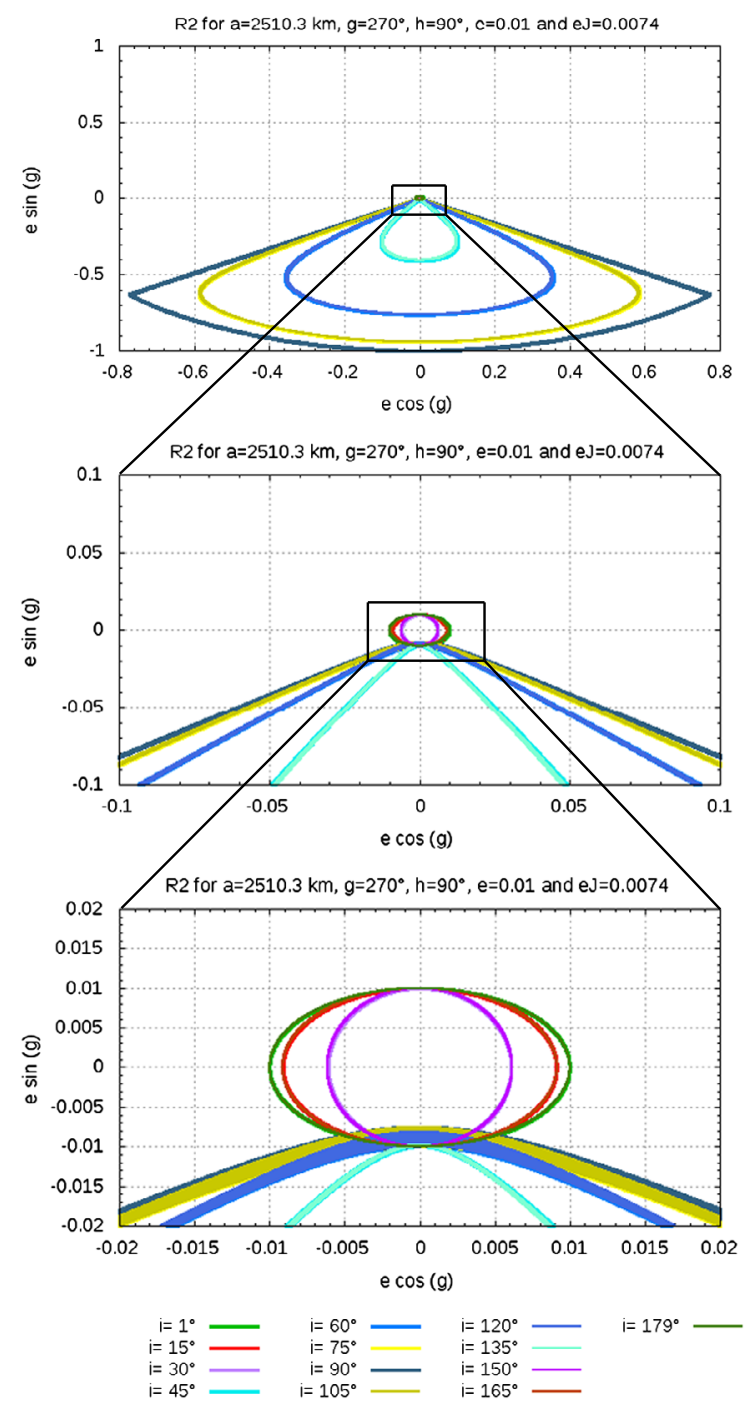

Fig. 4 Propagation of the components of the eccentricity vector considering different values for $i_{0}$ and with initial values $a_{0}=2510.3 \mathrm{~km}$, $e_{0}=0.01, h_{0}=90^{\circ}$ and $e_{J}=0.0074$. The effects are shown of $R_{2}$. Simulations for $g_{0}=270^{\circ}$ and numerical integration for 100 years 

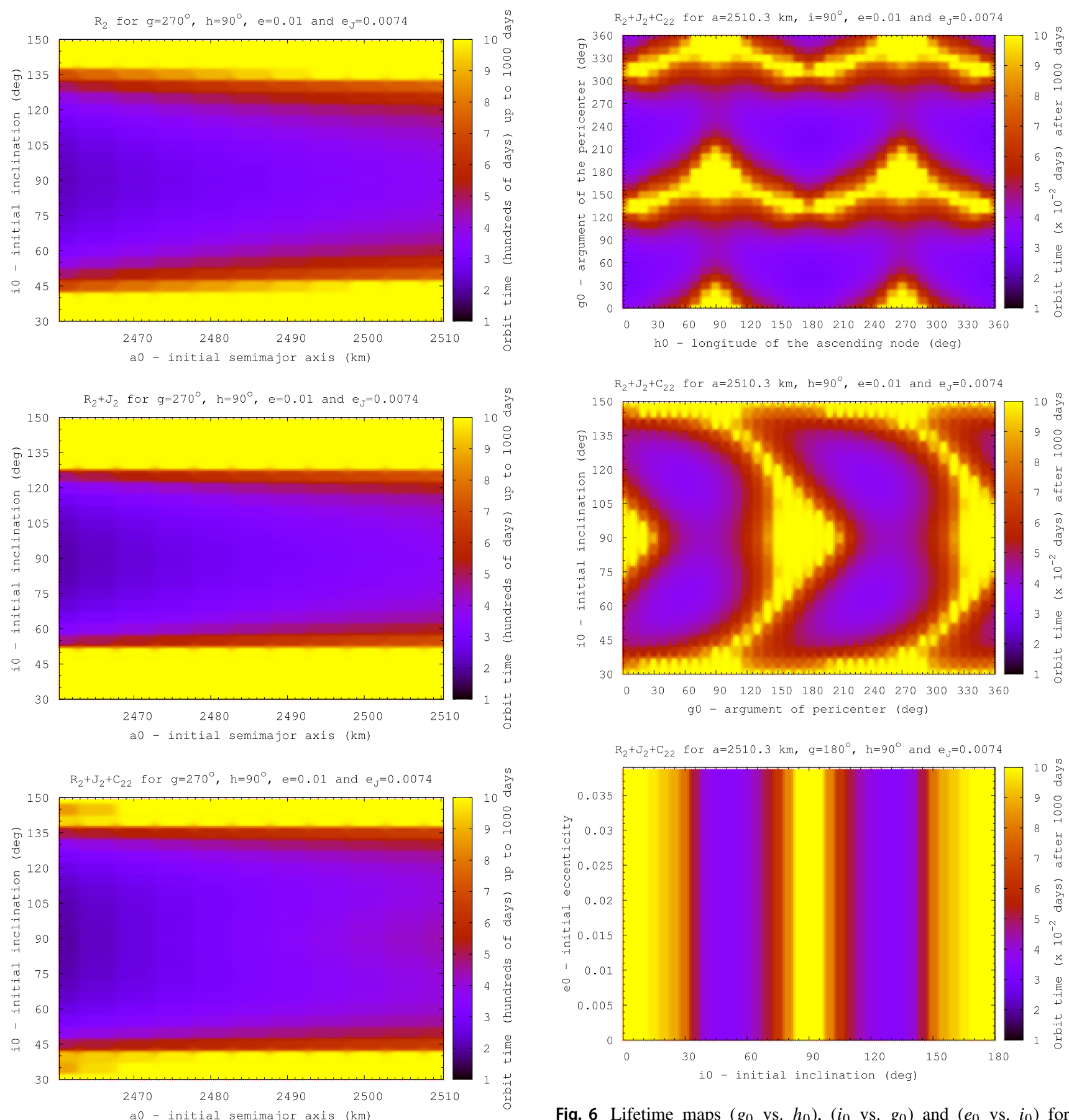

Fig. 5 Lifetime maps ( $i_{0}$ vs. $a_{0}$ ) as a function of semimajor axis and inclination considering the effects of $R_{2}$ and $R_{2}+J_{2}+C_{22}$. It is the real value for the eccentricity of Jupiter orbiting Callisto. Initial conditions: $e_{0}=0.01, g_{0}=270^{\circ}, h_{0}=90^{\circ}$ and $e_{J}=0.0074$. The vertical bar on the right gives the value of the lifetimes in hundreds of days for an integration of 1000 days. Each unit corresponds to one hundred days

Fig. 6 Lifetime maps $\left(g_{0}\right.$ vs. $\left.h_{0}\right),\left(i_{0}\right.$ vs. $\left.g_{0}\right)$ and $\left(e_{0}\right.$ vs. $\left.i_{0}\right)$ for $g_{0}=180^{\circ}, e_{J}=0.0074$ and considering the effects of $R_{2}+J_{2}+C_{22}$. The vertical bar on the right gives the value of the lifetimes in hundreds of days for an integration of 1000 days. Each unit corresponds to one hundred days 

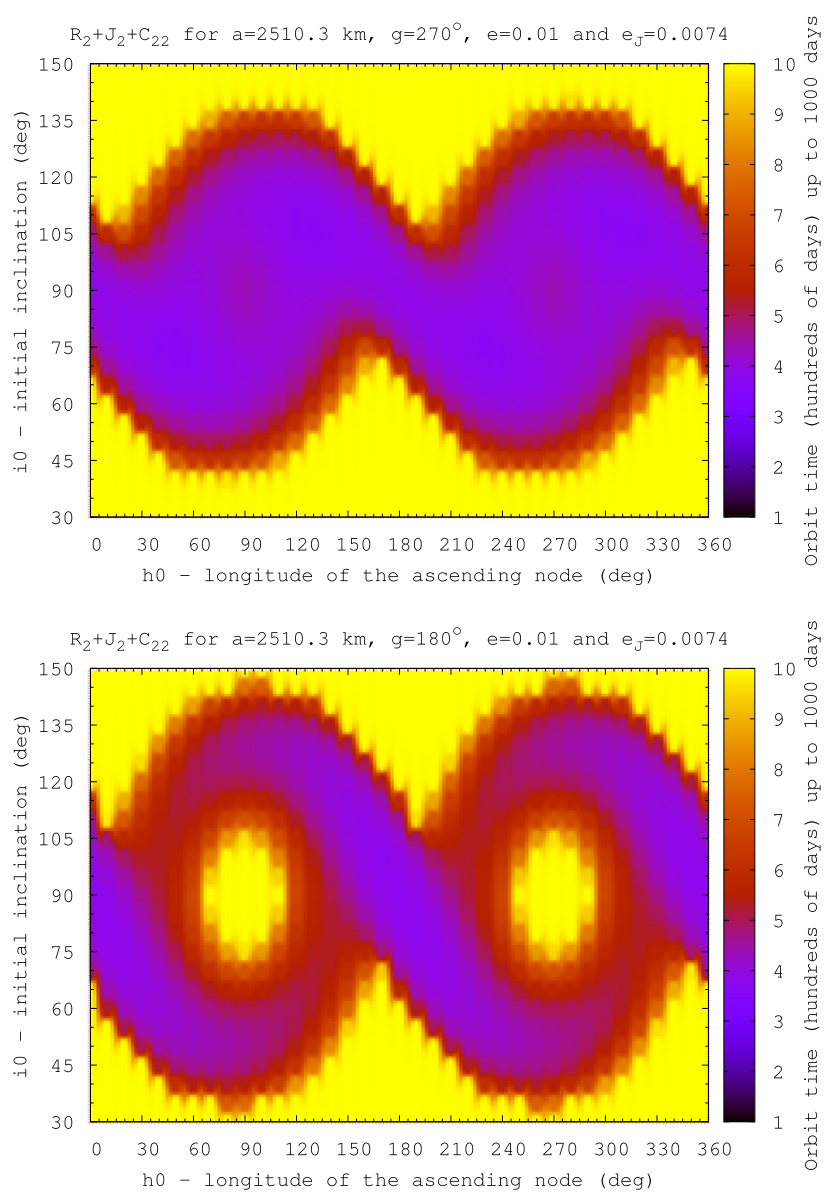

Fig. 7 Lifetime maps $\left(i_{0}\right.$ vs. $\left.h_{0}\right)$ for $e_{J}=0.0074$ and considering the effects of $R_{2}+J_{2}+C_{22}$. Simulations for $g_{0}=270^{\circ}$ and $180^{\circ}$. The vertical bar on the right gives the value of the lifetimes in hundreds of days for an integration of 1000 days. Each unit corresponds to one hundred days
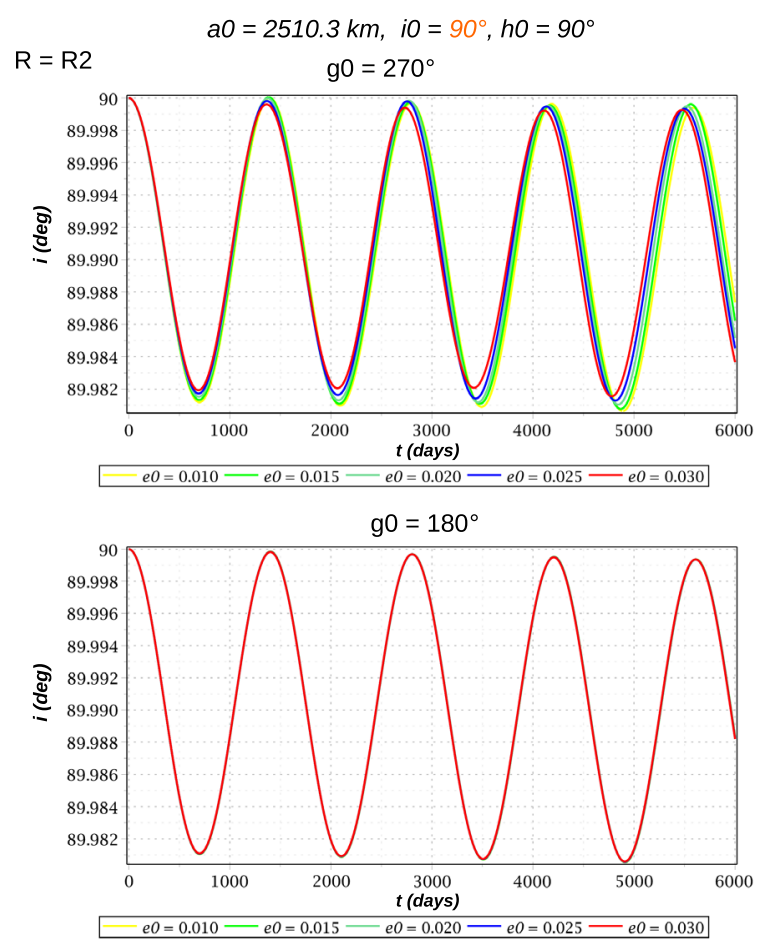

Fig. 8 Propagation of the eccentricity $(e)$, inclination $(i)$ and argument of the periapsis $(g)$ considering different values for the initial inclination $i_{0}$. Initial conditions: $a_{0}=2510.3 \mathrm{~km}, g_{0}=270^{\circ}, h_{0}=90^{\circ}$ and $e_{J}=0.0074$. The effects are shown that are due to $R_{2}$ 

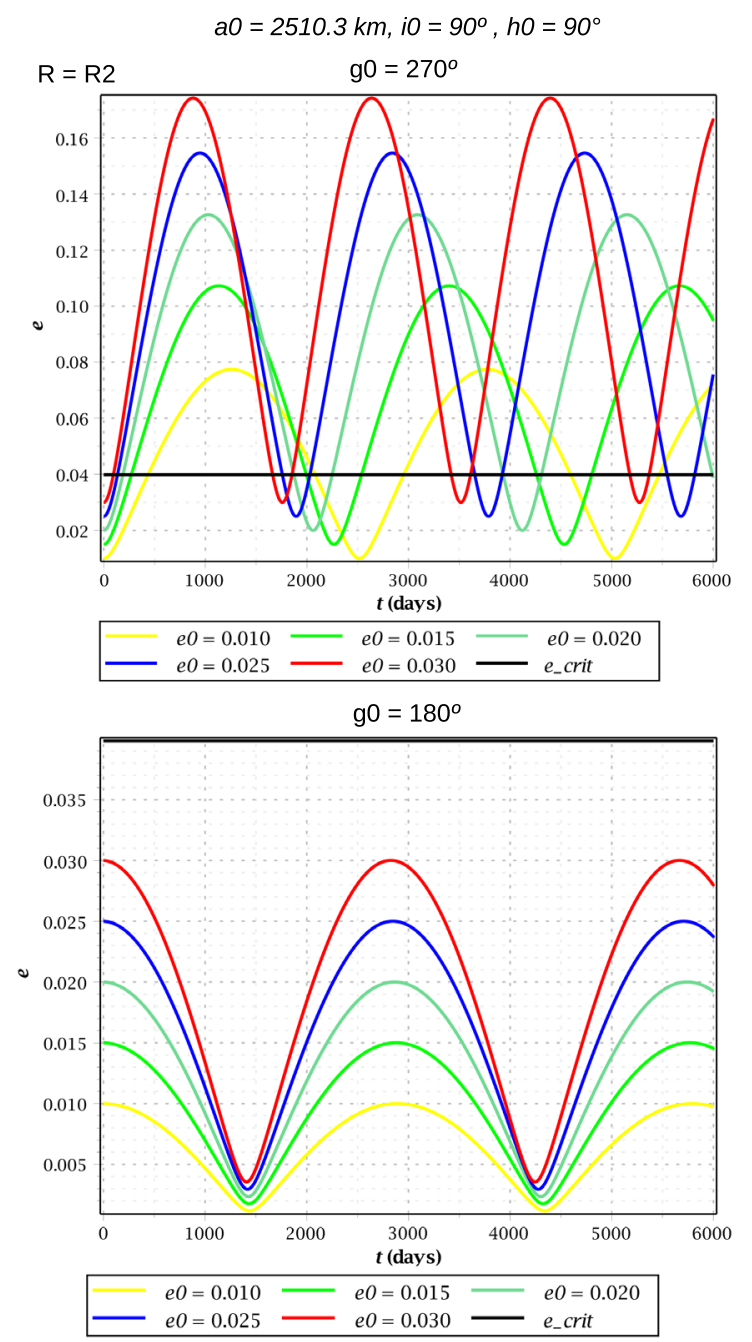

Fig. 9 Propagation of the eccentricity $(e)$ considering different values for the initial eccentricity $e_{0}$. Initial conditions: $a_{0}=2510.3 \mathrm{~km}$, $i_{0}=90^{\circ}, h_{0}=90^{\circ}$ and $e_{J}=0.0074$. The effects are shown that are due to $R_{2}$. Simulations for $g_{0}=270^{\circ}$ and $180^{\circ}$
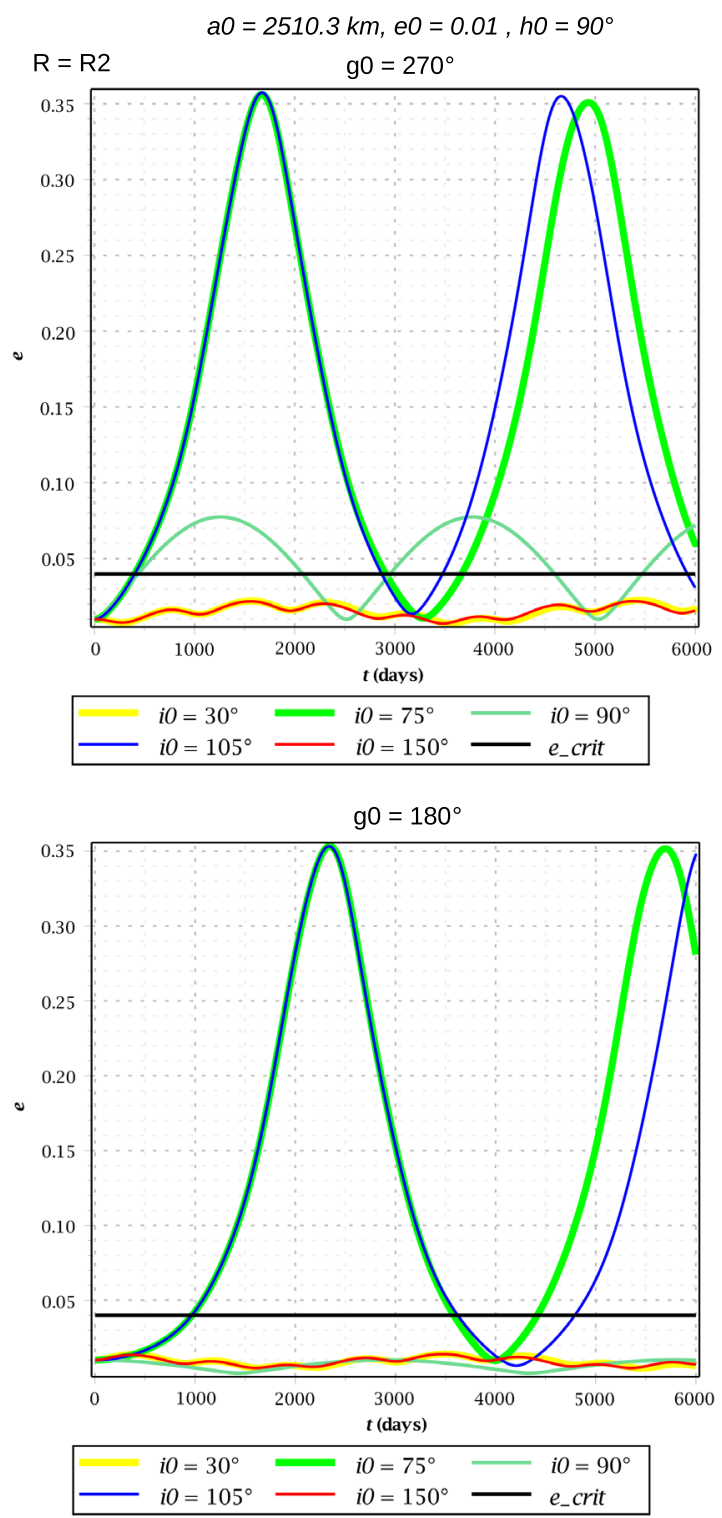

Fig. 10 Propagation of the eccentricity $(e)$ considering different values for the initial inclination $i_{0}$. Initial conditions: $a_{0}=2510.3 \mathrm{~km}$, $e_{0}=0.01, h_{0}=90^{\circ}$ and $e_{J}=0.0074$. The effects are shown that are due to $R_{2}$. Simulations for $g_{0}=270^{\circ}$ and $180^{\circ}$ 

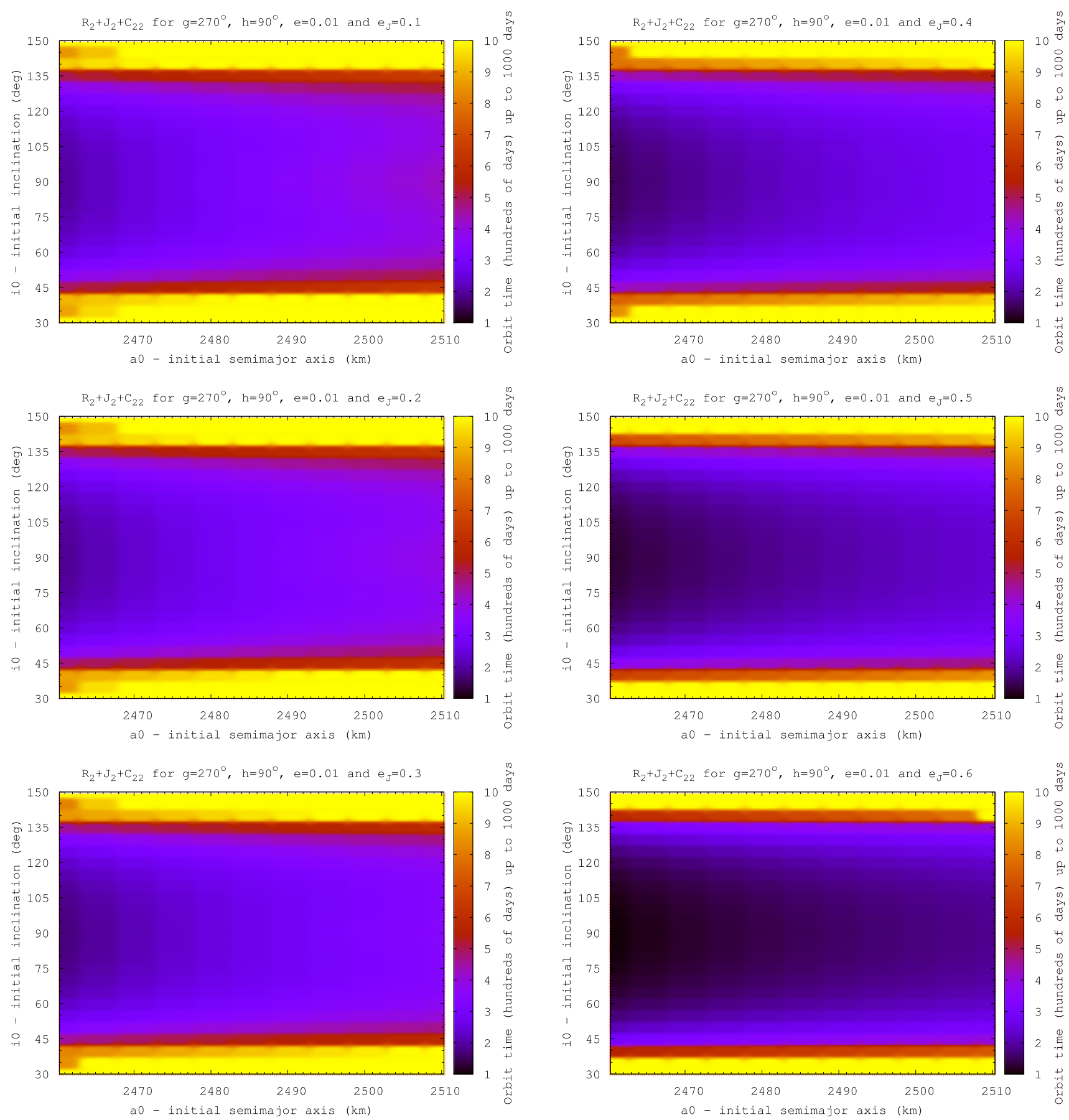

Fig. 11 Lifetime maps ( $i_{0}$ vs. $\left.a_{0}\right)$ for $e_{J}=0.1,0.2$ and 0.3. Disturbing effects: $R_{2}+J_{2}+C_{22}$. Initial conditions: $e_{0}=0.01, g_{0}=270^{\circ}$ and $h_{0}=90^{\circ}$. The vertical bar on the right gives the value of the lifetimes in hundreds of days for an integration of 1000 days. Each unit corresponds to one hundred days

Fig. 12 Lifetime maps ( $i_{0}$ vs. $\left.a_{0}\right)$ for $e_{J}=0.4,0.5$ and 0.6. Disturbing effects: $R_{2}+J_{2}+C_{22}$. Initial conditions: $e_{0}=0.01, g_{0}=270^{\circ}$ and $h_{0}=90^{\circ}$. The vertical bar on the right gives the value of the lifetimes in hundreds of days for an integration of 1000 days. Each unit corresponds to one hundred days 

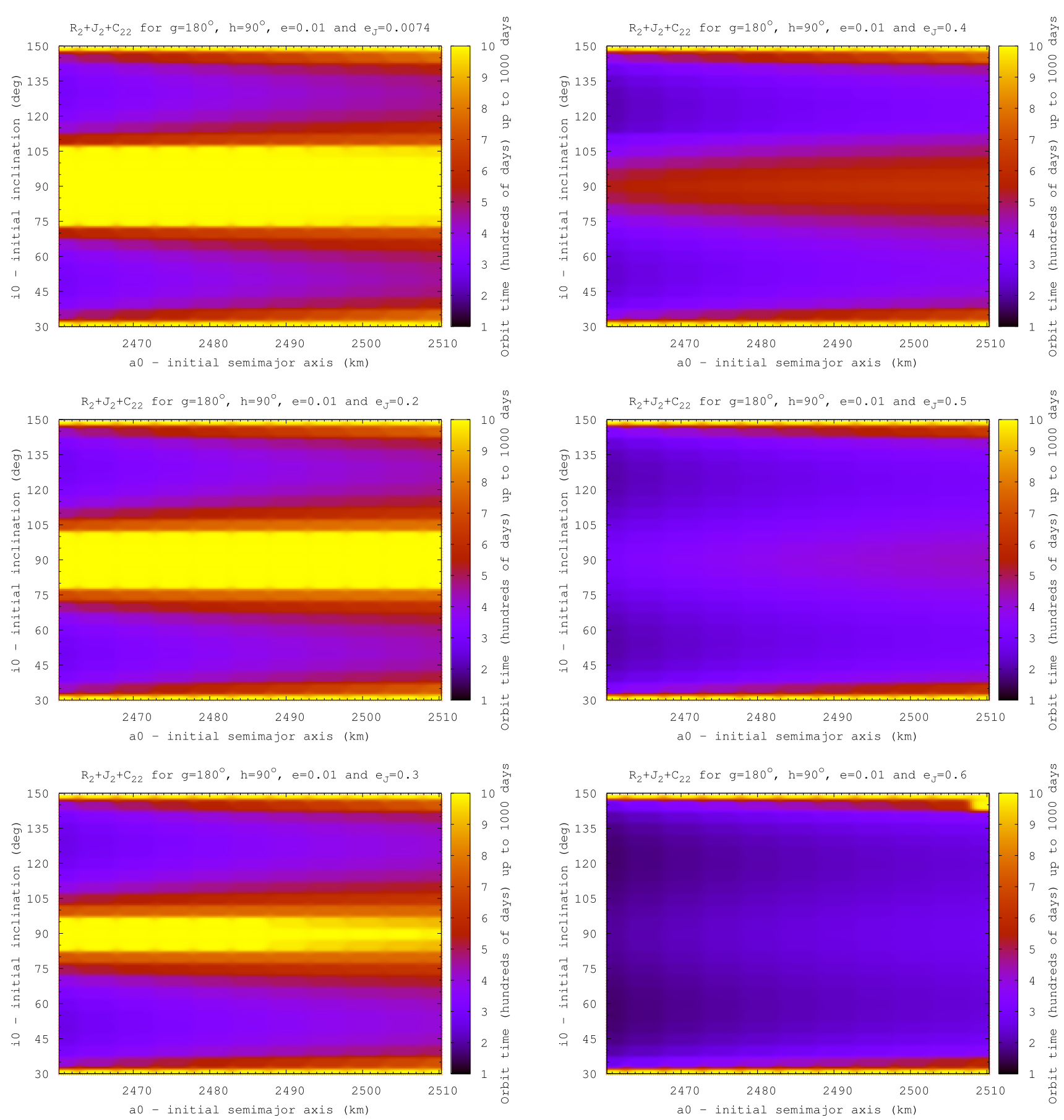

Fig. 13 Lifetime maps ( $i_{0}$ vs. $a_{0}$ ) for $e_{J}=0.0074,0.2$ and 0.3. Disturbing effects: $R_{2}+J_{2}+C_{22}$. Initial conditions: $e_{0}=0.01, g_{0}=180^{\circ}$ and $h_{0}=90^{\circ}$. The vertical bar on the right gives the value of the lifetimes in hundreds of days for an integration of 1000 days. Each unit corresponds to one hundred days

Fig. 14 Lifetime maps ( $i_{0}$ vs. $\left.a_{0}\right)$ for $e_{J}=0.4,0.5$ and 0.6. Disturbing effects: $R_{2}+J_{2}+C_{22}$. Initial conditions: $e_{0}=0.01, g_{0}=180^{\circ}$ and $h_{0}=90^{\circ}$. The vertical bar on the right gives the value of the lifetimes in hundreds of days for an integration of 1000 days. Each unit corresponds to hundred days 


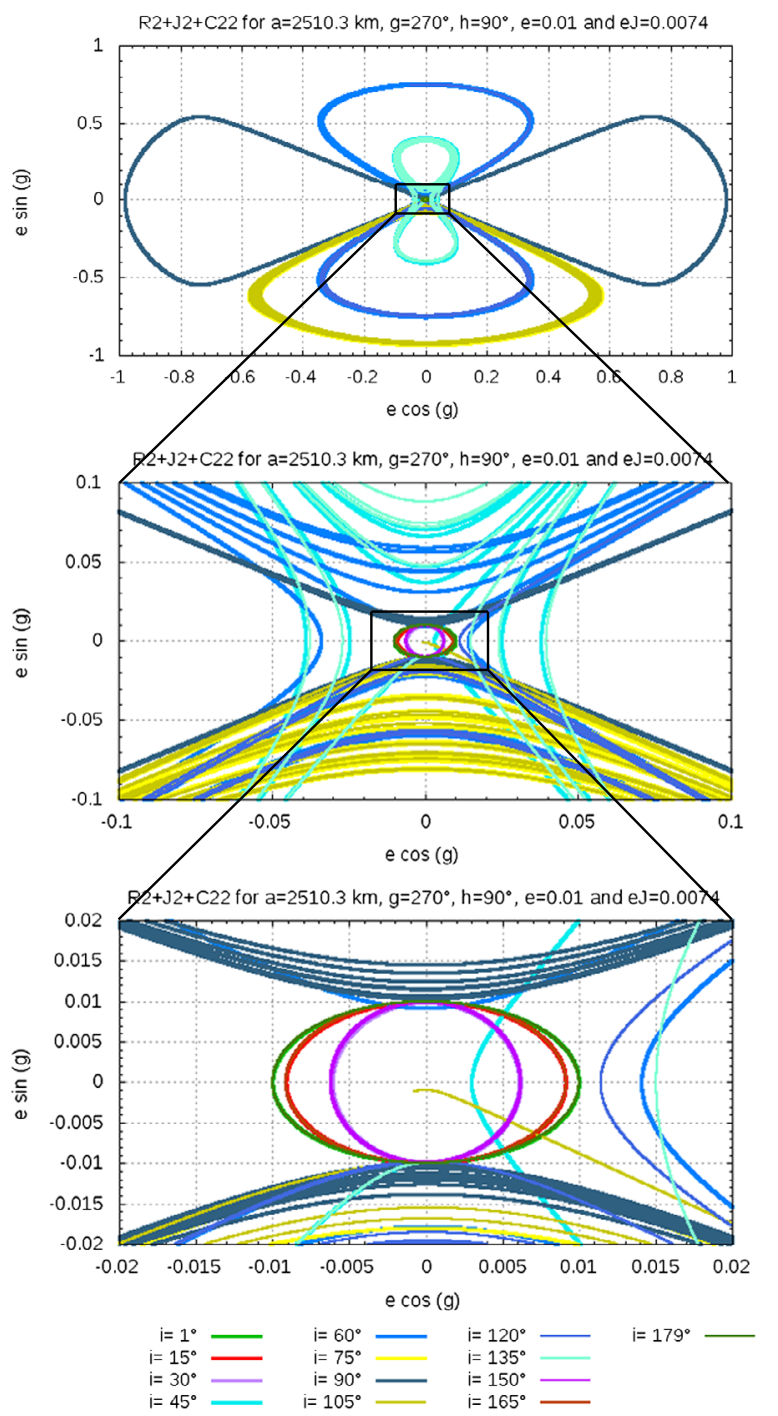

Fig. 15 Propagation of the components of the eccentricity vector considering different values for $i_{0}$ and with initial values $a_{0}=2510.3 \mathrm{~km}$, $e_{0}=0.01, h_{0}=9^{\circ}$ and $e_{J}=0.0074$. The effects are shown of $R_{2}+J_{2}+C_{22}$. Simulations for $g_{0}=270^{\circ}$ and numerical integration for 100 years

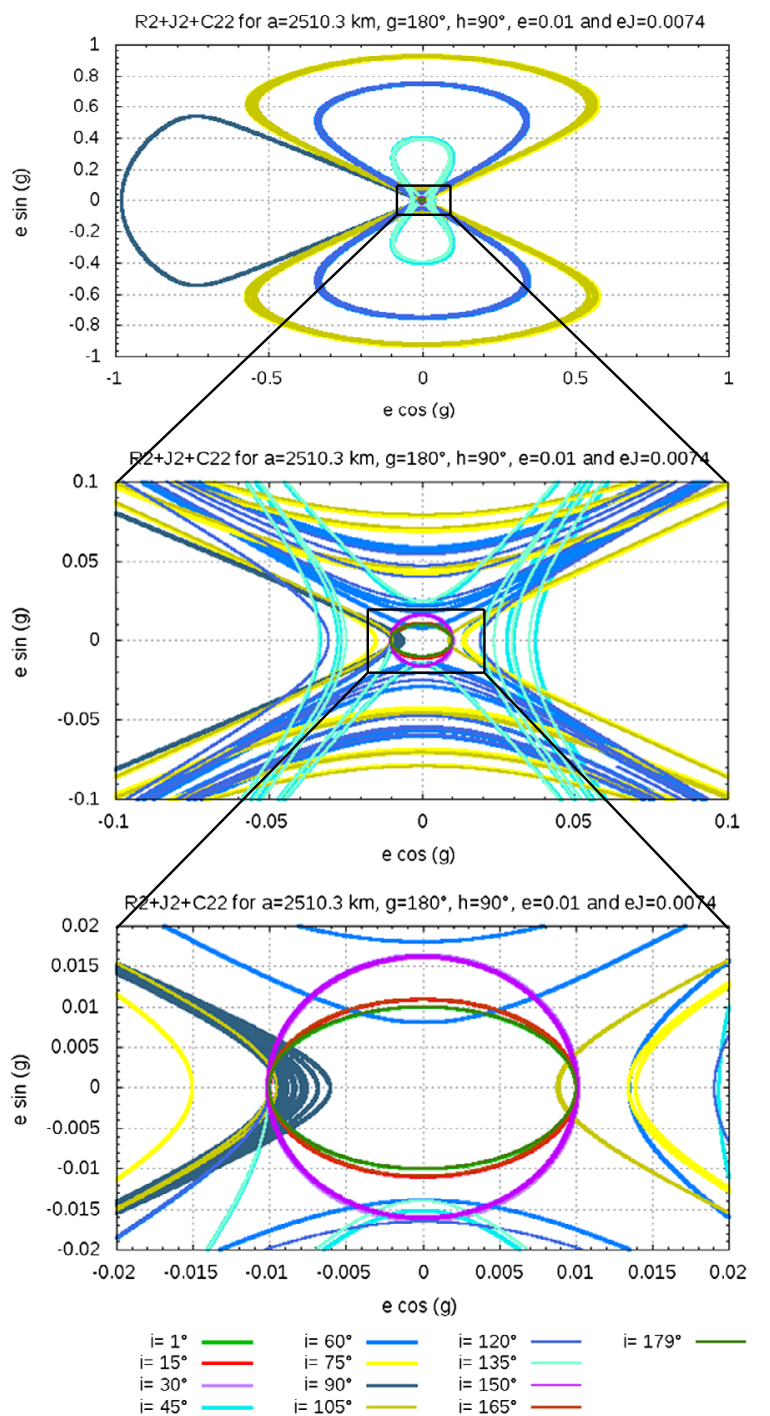

Fig. 16 Propagation of the components of the eccentricity vector considering different values for $i_{0}$ and with initial values $a_{0}=2510.3 \mathrm{~km}$, $e_{0}=0.01, h_{0}=9^{\circ}$ and $e_{J}=0.0074$. The effects are shown of $R_{2}+J_{2}+C_{22}$. Simulations for $g_{0}=180^{\circ}$ and numerical integration for 100 years 

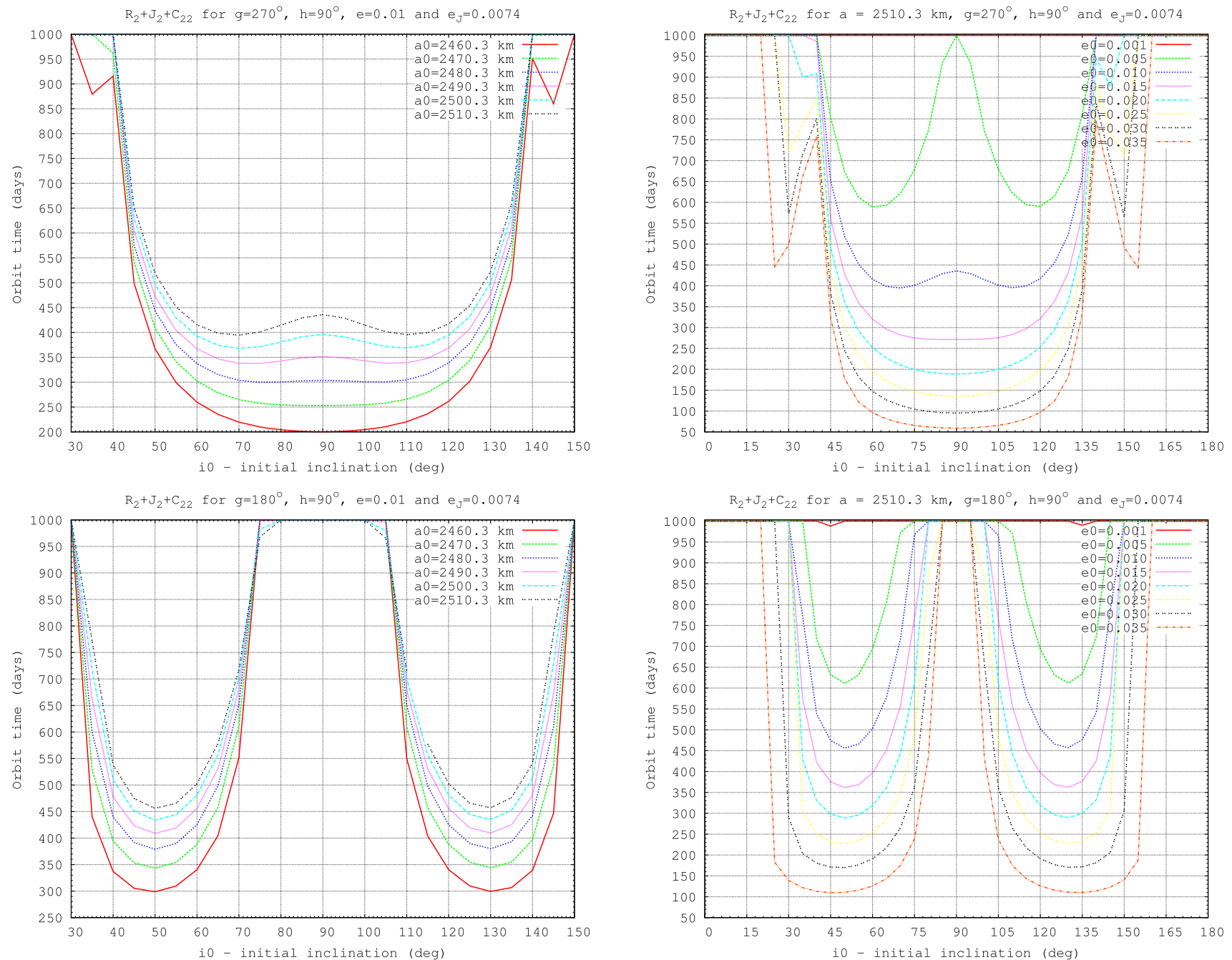

Fig. 17 Representation of orbit time vs. $i_{0}$ considering different values for $a_{0}$ and for $e_{0}=0.01$ and $e_{J}=0.0074$. The effects are shown of $R_{2}+J_{2}+C_{22}$. Simulations for $g_{0}=270^{\circ}$ and $180^{\circ}$

Fig. 18 Representation of orbit time vs. $i_{0}$ considering different values for $e_{0}$ and for $a_{0}=2510.3 \mathrm{~km}$ and $e_{J}=0.0074$. The effects are shown of $R_{2}+J_{2}+C_{22}$. Simulations for $g_{0}=270^{\circ}$ and $180^{\circ}$ 


\section{References}

Anderson, J.D., Jacobson, R.A., Lau, E.L., Moore, W.B., Schubert, G.: J. Geophys. Res. 106, 32963 (2001). doi:10.1029/2000JE001367

Araujo, R.A.N., Winter, O.C., Prado, A.F.B.A.: Mon. Not. R. Astron. Soc. 449, 4404 (2015). doi:10.1093/mnras/stv592. arXiv: 1503.07546

Blitzer, L.: Am. J. Phys. 27, 634 (1959). doi:10.1119/1.1934947

Boué, G., Laskar, J.: Icarus 201, 750 (2009)

Broucke, R.A.: J. Guid. Control Dyn. 26, 27 (2003). doi:10.2514/ 2.5041

Brouwer, D., Clemence, G.M.: Methods of Celestial Mechanics (1961)

Cardoso dos Santos, J., Carvalho, J.P.S., Vilhena De Moraes, R., Prado, A.F.B.A.: In: International Astronautical Congress, Jerusalem, Israel, 12-16 October (2015). IAC paper C1.IP.4

Carvalho, J.P.S.: Orbital evolution of a solar sail around a planet. In: Proceeding Series of the Brazilian Society of Applied and Computational Mathematics, vol. 4 (2016). doi:10.5540/ 03.2016.004.01.0017

Carvalho, J.P.S., Vilhena de Moraes, R., Prado, A.F.B.A.: Celest. Mech. Dyn. Astron. 108, 371 (2010). doi:10.1007/s10569010-9310-6

Carvalho, J.P.S., Mourão, D.C., Elipe, A., Vilhena De Moraes, R., Prado, A.F.B.A.: Int. J. Bifurc. Chaos Appl. Sci. Eng. 22, 1250240 (2012a). doi:10.1142/S0218127412502409

Carvalho, J.P.S., Elipe, A., Vilhena De Moraes, R., Prado, A.F.B.A.: Adv. Space Res. 49, 994 (2012b). doi:10.1016/j.asr.2011.11.036

Carvalho, J.P.S., de Moraes, R.V., Prado, A.F.B.A., Mourão, D.C., Winter, O.C.: Comput. Appl. Math. 35, 847 (2016a). doi:10.1007/ s40314-015-0270-z

Carvalho, J.P.S., Mourão, D.C., de Moraes, R.V., Prado, A.F.B.A., Winter, O.C.: Celest. Mech. Dyn. Astron. 124, 73 (2016b). doi:10.1007/s10569-015-9650-3

Carvalho, J.P.S., Cardoso dos Santos, J., Prado, A.F.B.A., Vilhena De Moraes, R.: Some characteristics of orbits for a spacecraft around Mercury, Comput. Appl. Math. (2017). doi:10.1007/s40314-017-0525-y

Condoleo, E., Cinelli, M., Ortore, E., Circi, C.: J. Guid. Control Dyn. 39, 2264 (2016). doi:10.2514/1.G000455

Domingos, R.C., Prado, A.F.B.A., Gomes, V.M.: Math. Probl. Eng. 2014, 359845 (2014). doi:10.1155/2014/359845

Domingos, R.C., Vilhena De Moraes, R., Prado, A.F.B.A.: Math. Probl. Eng. 2008, 763654 (2008). doi:10.1155/2008/763654

ESA: Juice Definition Study Report (red Book) (2014). http:// sci.esa.int/juice/54994-juice-definition-study-report/

Fang, J., Margot, J., Brozovic, M., Nolan, M.C., Benner, L.A.M., Taylor, P.A.: Astron. J. 141, 154 (2011). doi:10.1088/0004-6256/ $141 / 5 / 154$

Farago, F., Laskar, J.: Mon. Not. R. Astron. Soc. 401, 1189 (2010). doi:10.1111/j.1365-2966.2009.15711.x. arXiv:0909.2287
Ferrer, S., Osacar, C.: Celest. Mech. Dyn. Astron. 58, 245 (1994). doi:10.1007/BF00691977

Giacaglia, G.E.O.: SAO Special Report, 352 (1973)

Giacaglia, G.E.O., Murphy, J.P., Felsentreger, T.L.: Celest. Mech. 3, 3 (1970). doi:10.1007/BF01230432

Giuliatti Winter, S.M., Winter, O.C., Vieira Neto, E., Sfair, R.: Mon. Not. R. Astron. Soc. 430, 1892 (2013). doi:10.1093/mnras/stt015

Gomes, V., Domingos, R.C.: Comput. Appl. Math. 35, 653 (2016). doi: $10.1007 / \mathrm{s} 40314-015-0258-8$

Hough, M.E.: Celest. Mech. 25, 111 (1981). doi:10.1007/BF01230514

Kaula, W.M.: Astron. J. 67, 300 (1962). doi:10.1086/108729

Kovalevsky, J. (ed.): Introduction to Celestial Mechanics. Astrophys. Space Sci. Library, vol. 7 (1967). doi:10.1007/978-94011-7545-6

Kozai, Y.: On the effects of the sun and moon upon the motion of a close earth satellite. Technical report (1959)

Kozai, Y.: Astron. J. 67, 591 (1962). doi:10.1086/108790

Lara, M., Russell, R.: In: 2006 AAS/AIAA SpaceFlight Mechanics Meeting, Tampa, Florida, January 22-26 (2006). http:// hdl.handle.net/2014/38715

Lidov, M.L.: Planet. Space Sci. 9, 719 (1962). doi:10.1016/00320633(62)90129-0

Liu, X., Baoyin, H., Ma, X.: Astrophys. Space Sci. 339, 295 (2012). doi:10.1007/s10509-012-1015-8. arXiv:1203.1770

Meyer, K.W., Buglia, J.J., Desai, P.N.: NASA STI/Recon Technical Report N 94 (1994)

Murray, C.D., Dermott, S.F.: Solar System Dynamics (2000)

Musen, P., Bailie, A., Upton, E.: NASA Spec. Publ. 54, 24 (1965)

Naoz, S.: ArXiv e-prints (2016). arXiv:1601.07175

Naoz, S., Farr, W.M., Lithwick, Y., Rasio, F.A., Teyssandier, J.: Nature 473, 187 (2011). doi:10.1038/nature10076. arXiv:1011.2501

Naoz, S., Li, G., Zanardi, M., de Elía, G.C., Di Sisto, R.P.: ArXiv e-prints (2017). arXiv:1701.03795

NASA: Mission to Europa (Acessed: January/2017). https://www. nasa.gov/europa/

Ortiz, J.L., Santos-Sanz, P., Sicardy, B., et al.: Nature 550, 219 (2017). doi:10.1038/nature24051

Paskowitz, M.E., Scheeres, D.J.: J. Guid. Control Dyn. 29, 1147 (2006). doi:10.2514/1.19464

Prado, A.F.B.A.: J. Guid. Control Dyn. 26, 33 (2003). doi:10.2514/ 2.5042

Prado, A.F.B.A.: Adv. Space Res. 53, 877 (2014). doi:10.1016/ j.asr.2013.12.034

Scheeres, D.J., Guman, M.D., Villac, B.F.: J. Guid. Control Dyn. 24, 778 (2001). doi: $10.2514 / 2.4778$

Sehnal, L.: Bull. Astron. Inst. Czechoslov. 11, 90 (1960)

Tresaco, E., Elipe, A., Carvalho, J.P.S.: J. Guid. Control Dyn. 39(7), 1659 (2016). doi:10.2514/1.G001510

Vilhena De Moraes, R., Costa, M.L.G.T.X., Carvalho, J.P.S., Prado, A.F.B.A.: In: International Astronautical Congress, Guadalajara, Mexico, 26-30 September (2016). IAC paper C1,6,10,x35324 